\title{
Fixed Points in Menger Space for Compatible Mappings of Type
}

( $\beta)$

\section{H. Badshah}

School of Studies in Mathematics, Department of Mathematics,

Vikram University,

Ujjain (M.P.), India
Suman Jain

Govt. College,

Kalapipal (M.P.) India
Subhash Mandloi

School of Studies in Mathematics,

Vikram University,

Ujjain (M.P.), India

\begin{abstract}
In this paper, the concept of compatibility of type $(\beta)$ and occasionally weak compatibility in Menger space has been applied to prove a common fixed point theorem for six self maps which generalizes the result of Pant et. al. [7].
\end{abstract}

Keywords and Phrases: Menger space, Common fixed points, Compatible maps, Compatible maps of type ( $\beta)$ and Occasionally Weak compatibility.

\section{INTRODUCTION}

In 1942, K. Menger [5] introduced the notion of probabilistic metric space (briefly, PM-space) as a generalization of metric space. Such a probabilistic generalization of metric spaces appears to be well adapted for the investigation of physical quantities and physiological thresholds. It is also of fundamental importance in probabilistic functional analysis. The development of fixed point theory in PM-spaces was due to Schweizer and Sklar [8]. Sehgal and Bharucha-Reid [9] obtained a generalization of Banach Contraction Principle on a complete Menger space which is a milestone in developing fixed-point theory in Menger space.

Recently, Jungck and Rhoades [4] termed a pair of self maps to be coincidentally commuting or equivalently weakly compatible if they commute at their coincidence points. Sessa [10] initiated the tradition of improving commutativity in fixed-point theorems by introducing the notion of weak commuting maps in metric spaces. Jungck [3] soon enlarged this concept to compatible maps. The notion of compatible mapping in a Menger space has been introduced by Mishra [6].

Recently, in 2013, Jain et. al. [2] proved a common fixed point theorem using the concept of semicompatibility and occasionally weak compatibility in Menger space.

In this paper a fixed point theorem for six self maps has been proved using the concept of occasionally weak compatibility and compatibility of type $(\beta)$ which generalizes the result of Pant et.al. [7]. We also cited an example.

\section{Preliminaries}

Definition 2.1.[6] A mapping $F: R \rightarrow \mathrm{R}^{+}$is called a distribution if it is non-decreasing left continuous with

$$
\inf \{\mathcal{F}(\mathrm{t}) \mid \mathrm{t} \in \mathrm{R}\}=0 \quad \text { and } \quad \sup \{\mathcal{F}(\mathrm{t}) \mid \mathrm{t} \in \mathrm{R}\}=1 .
$$

We shall denote by $\mathrm{L}$ the set of all distribution functions while $\mathrm{H}$ will always denote the specific distribution function defined by

$$
\mathrm{H}(\mathrm{t})= \begin{cases}0, & \mathrm{t} \leq 0 \\ 1, & \mathrm{t}>0\end{cases}
$$


Definition 2.2. [6] A mapping $\mathrm{t}:[0,1] \times[0,1] \rightarrow[0,1]$ is called a $t$-norm if it satisfies the following conditions :

$$
\begin{aligned}
& \mathrm{t}(\mathrm{a}, 1)=\mathrm{a}, \quad \mathrm{t}(0,0)=0 \\
& \mathrm{t}(\mathrm{a}, \mathrm{b})=\mathrm{t}(\mathrm{b}, \mathrm{a}) ; \\
& \mathrm{t}(\mathrm{c}, \mathrm{d}) \geq \mathrm{t}(\mathrm{a}, \mathrm{b}) ; \quad \text { for } \mathrm{c} \geq \mathrm{a}, \mathrm{d} \geq \mathrm{b}, \\
& \mathrm{t}(\mathrm{t}(\mathrm{a}, \mathrm{b}), \mathrm{c})=\mathrm{t}(\mathrm{a}, \mathrm{t}(\mathrm{b}, \mathrm{c})) \text { for all } \mathrm{a}, \mathrm{b}, \mathrm{c}, \mathrm{d} \in[0,1] .
\end{aligned}
$$

Definition 2.3. [6] A probabilistic metric space (PM-space) is an ordered pair (X, F) consisting of a non-empty set $\mathrm{X}$ and a function $F: \mathrm{X} \times \mathrm{X} \rightarrow \mathrm{L}$, where $\mathrm{L}$ is the collection of all distribution functions and the value of $F$ at $(\mathrm{u}, \mathrm{v}) \in \mathrm{X} \times \mathrm{X}$ is represented by $\mathrm{F}_{\mathrm{u}, \mathrm{v}}$. The function $\mathrm{F}_{\mathrm{u}, \mathrm{v}}$ assumed to satisfy the following conditions:

$(\mathrm{PM}-1) \mathrm{F}_{\mathrm{u}, \mathrm{v}}(\mathrm{x})=1$, for all $\mathrm{x}>0$, if and only if $\mathrm{u}=\mathrm{v}$;

$(\mathrm{PM}-2) \mathrm{F}_{\mathrm{u}, \mathrm{v}}(0)=0$;

(PM-3) $\mathrm{F}_{\mathrm{u}, \mathrm{v}}=\mathrm{F}_{\mathrm{v}, \mathrm{u}}$;

(PM-4) If $\mathrm{F}_{\mathrm{u}, \mathrm{v}}(\mathrm{x})=1$ and $\mathrm{F}_{\mathrm{v}, \mathrm{w}}(\mathrm{y})=1$ then $\mathrm{F}_{\mathrm{u}, \mathrm{w}}(\mathrm{x}+\mathrm{y})=1, \quad$ for all $\mathrm{u}, \mathrm{v}, \mathrm{w} \in \mathrm{X}$ and $\mathrm{x}, \mathrm{y}>0$.

Definition 2.4. [6] A Menger space is a triplet $(\mathrm{X}, \mathcal{F}, \mathrm{t})$ where $(\mathrm{X}, \mathcal{F})$ is a $\mathrm{PM}$-space and t is a t-norm such that the inequality

(PM-5) $\mathrm{F}_{\mathrm{u}, \mathrm{w}}(\mathrm{x}+\mathrm{y}) \geq \mathrm{t}\left\{\mathrm{F}_{\mathrm{u}, \mathrm{v}}(\mathrm{x}), \mathrm{F}_{\mathrm{v}, \mathrm{w}}(\mathrm{y})\right\}$, for all $\mathrm{u}, \mathrm{v}, \mathrm{w} \in X, \mathrm{x}, \mathrm{y} \geq 0$.

Definition 2.5. [6] A sequence $\left\{\mathrm{x}_{\mathrm{n}}\right\}$ in a Menger space $(\mathrm{X}, \boldsymbol{F}, \mathrm{t})$ is said to be convergent and converges to a point $\mathrm{x}$ in $\mathrm{X}$ if and only if for each $\varepsilon>0$ and $\lambda>0$, there is an integer $\mathrm{M}(\varepsilon, \lambda)$ such that

$$
\mathrm{F}_{\mathrm{X}_{\mathrm{n}}, \mathrm{X}}(\varepsilon)>1-\lambda \quad \text { for all } \mathrm{n} \geq \mathrm{M}(\varepsilon, \lambda) \text {. }
$$

Further the sequence $\left\{\mathrm{x}_{\mathrm{n}}\right\}$ is said to be Cauchy sequence if for $\varepsilon>0$ and $\lambda>0$, there is an integer $\mathrm{M}(\varepsilon, \lambda)$ such that

$$
\mathrm{F}_{\mathrm{X}_{\mathrm{n}}, \mathrm{x}_{\mathrm{m}}}(\varepsilon)>1-\lambda \quad \text { for all } \mathrm{m}, \mathrm{n} \geq \mathrm{M}(\varepsilon, \lambda) \text {. }
$$

A Menger PM-space $(\mathrm{X}, \mathcal{F}, \mathrm{t})$ is said to be complete if every Cauchy sequence in $\mathrm{X}$ converges to a point in $\mathrm{X}$.

A complete metric space can be treated as a complete Menger space in the following way:

Proposition 2.1. [2] If ( $\mathrm{X}, \mathrm{d})$ is a metric space then the metric $\mathrm{d}$ induces mappings $F: X \times X \rightarrow L$, defined by $\mathrm{F}_{\mathrm{p}, \mathrm{q}}(\mathrm{x})=\mathrm{H}(\mathrm{x}-\mathrm{d}(\mathrm{p}, \mathrm{q})), \mathrm{p}, \mathrm{q} \in \mathrm{X}$, where

$$
\mathrm{H}(\mathrm{k})=0 \text {, for } \mathrm{k} \leq 0 \text { and } \mathrm{H}(\mathrm{k})=1 \text {, for } \mathrm{k}>0 \text {. }
$$

Further if, $\mathrm{t}:[0,1] \times[0,1] \rightarrow[0,1]$ is defined by $\mathrm{t}(\mathrm{a}, \mathrm{b})=\min \{\mathrm{a}, \mathrm{b}\}$. Then $(X, F, \mathrm{t})$ is $\mathrm{a}$ Menger space. It is complete if $(\mathrm{X}, \mathrm{d})$ is complete.

The space $(\mathrm{X}, \mathcal{F}, \mathrm{t})$ so obtained is called the induced Menger space.

Definition 2.6. [2] Self mappings $A$ and $S$ of a Menger space $(X, F, t)$ are said to be weak compatible if they commute at their coincidence points i.e. $A x=S x$ for $x \in X$ implies $A S x=S A x$.

Definition 2.7. [6] Self mappings $A$ and $S$ of a Menger space $(X, F, t)$ are said to be compatible if $\mathrm{F}_{\mathrm{ASx}_{\mathrm{n}}, \mathrm{SAx}_{\mathrm{n}}}(\mathrm{x}) \rightarrow 1$ for all $\mathrm{x}>0$, whenever $\left\{\mathrm{x}_{\mathrm{n}}\right\}$ is a sequence in $\mathrm{X}$ such that $\mathrm{Ax}_{\mathrm{n}}, \mathrm{Sx}_{\mathrm{n}} \rightarrow \mathrm{u}$ for some $\mathrm{u}$ in $\mathrm{X}$, as $\mathrm{n} \rightarrow \infty$. 
Definition 2.8. [1] Self maps $\mathrm{S}$ and $\mathrm{T}$ of a Menger space $(\mathrm{X}, \mathcal{F}, \mathrm{t})$ are said to be compatible of type $(\beta)$ if $\mathrm{FSSx}_{\mathrm{n}}, \operatorname{TTX}_{\mathrm{n}}(\mathrm{x}) \rightarrow 1$ for all $\mathrm{x}>0$, whenever $\left\{\mathrm{x}_{\mathrm{n}}\right\}$ is a sequence in $\mathrm{X}$ such that $\mathrm{Sx}_{\mathrm{n}}, \mathrm{Tx}_{\mathrm{n}} \rightarrow \mathrm{u}$ for some $\mathrm{u}$ in $\mathrm{X}$, as $\mathrm{n} \rightarrow \infty$.

Definition 2.9. [7] Self maps $S$ and $T$ of a Menger space $(X, F, t)$ are said to be semi-compatible if $\mathrm{F}_{\mathrm{STx}_{\mathrm{n}}, \mathrm{Tu}}(\mathrm{x}) \rightarrow 1$ for all $\mathrm{x}>0$, whenever $\left\{\mathrm{x}_{\mathrm{n}}\right\}$ is a sequence in $\mathrm{X}$ such that $\mathrm{Sx}_{\mathrm{n}}, \mathrm{Tx}_{\mathrm{n}} \rightarrow \mathrm{u}$ for some $\mathrm{u}$ in $\mathrm{X}$, as $\mathrm{n} \rightarrow \infty$.

Definition 2.10. [2] Self maps $A$ and $S$ of a Menger space $(X, F, t)$ are said to be occasionally weakly compatible (owc) if and only if there is a point $\mathrm{X}$ in $\mathrm{X}$ which is coincidence point of $\mathrm{A}$ and $\mathrm{S}$ at which $\mathrm{A}$ and $\mathrm{S}$ commute.

Now, we give an example of pair of self maps (I, L) which are compatible of type (P) but notsemi-compatible.

Example 2.1. Let $(X, d)$ be a metric space where $X=[0,1]$ and $(X, F, t)$ be the induced Menger space with $F_{x, y}=\frac{t}{t+d(x, y)}$ for all $t>0$.

Define self maps I and $\mathrm{L}$ as follows :

$$
I(x)=x \text { for all } x \in X \text { and } L(x)=\left\{\begin{array}{lll}
x, & \text { if } & 0 \leq x<\frac{1}{2} \\
1, & \text { if } \quad \frac{1}{2} \leq x \leq 1 .
\end{array}\right.
$$

Taking $\mathrm{x}_{\mathrm{n}}=\frac{1}{2}-\frac{1}{\mathrm{n}}$, we get $\mathrm{Ix}_{\mathrm{n}}=\mathrm{x}_{\mathrm{n}}=\frac{1}{2}-\frac{1}{\mathrm{n}}$ and $\mathrm{Lx}_{\mathrm{n}}=\frac{1}{2}-\frac{1}{\mathrm{n}}$.

Thus, $\mathrm{Lx}_{\mathrm{n}} \rightarrow \frac{1}{2} \quad$ as $\mathrm{n} \rightarrow \infty$ and $\mathrm{Ix}_{\mathrm{n}} \rightarrow \frac{1}{2}$, as $\mathrm{n} \rightarrow \infty$.

Hence, $x=\frac{1}{2}$

Since $\operatorname{Lx}_{\mathrm{n}}=\frac{1}{2}-\frac{1}{\mathrm{n}}$

Therefore, $\operatorname{IIx}_{\mathrm{n}}=\mathrm{I}\left(\frac{1}{2}-\frac{1}{\mathrm{n}}\right)=\frac{1}{2}-\frac{1}{\mathrm{n}}$

and $\quad \operatorname{LLx}_{\mathrm{n}}=\mathrm{L}\left(\frac{1}{2}-\frac{1}{\mathrm{n}}\right)=\frac{1}{2}-\frac{1}{\mathrm{n}}$.

Consider $\lim _{n \rightarrow \infty} F_{I x_{n}, \operatorname{LLx} x_{n}}(t)=\lim _{n \rightarrow \infty} F_{\frac{1}{2}-\frac{1}{n}, \frac{1}{2}-\frac{1}{n}}(t)=1$ for $t>0$.

Therefore, by definition, (I, L) is compatible mapping of type $(\beta)$.

Now, $\lim _{n \rightarrow \infty} F_{I L x_{n}, L x}(t)=\lim _{n \rightarrow \infty} F_{\frac{1}{2}-\frac{1}{n}, 1}(t)<1$ for $t>0$.

Therefore, (I, L) is not semi-compatible mapping. Thus the pair (I, L) of self maps is compatible of type $(\beta)$ but not semi-compatible.

Remark 2.2. In view of above example, it follows that the concept of compatible maps of type $(\beta)$ is more general than that of semi-compatible maps. 
Example 2.2. Let $(\mathrm{X}, \mathrm{d})$ be a metric space where $\mathrm{X} \in \mathrm{R}^{+}$and $(\mathrm{X}, \boldsymbol{F}, \mathrm{t})$ be the induced Menger space with $F_{x, y}=\frac{t}{t+d(x, y)}$ for all $t>0, x, y \in X$.

Define self maps $\mathrm{S}$ and $\mathrm{T}$ as follows :

$$
S(x)=\left\{\begin{array}{ccc}
\frac{2}{x^{2}}, & \text { if } & x \neq 0 \\
1, & \text { if } & x=0
\end{array} \text { and } T(x)=\left\{\begin{array}{ccc}
\frac{2}{x}, & \text { if } & x \neq 0 \\
1, & \text { if } & x=0
\end{array}\right.\right.
$$

Taking $\mathrm{x}_{\mathrm{n}}=\mathrm{n}$, we get

$$
\lim _{\mathrm{n} \rightarrow \infty} \mathrm{F}_{\mathrm{SSx}_{\mathrm{n}}, \mathrm{TTx}_{\mathrm{n}}}(\mathrm{t}) \neq 1 \text { for } \mathrm{t}>0 \text {. }
$$

Hence, the pair $(S, T)$ is not compatible of type $(\beta)$.

Also, 0 and 1 are coincidence points of $S$ and $T$ but $(S, T)$ commute only at point 0 .

Thus, the pair $(\mathrm{S}, \mathrm{T})$ is occasionally weakly compatible.

Remark 2.1. In view of example 2.2 , the concept of occasionally weakly compatible is more general than that of compatibility of type $(\beta)$ and weak compatibility.

Lemma 2.1. [11] Let $\left\{\mathrm{x}_{\mathrm{n}}\right\}$ be a sequence in a Menger space $(X, \mathcal{F}, \mathrm{t})$ with continuous t-norms $t$ and $t(a, a) \geq a$. If there exists a constant $k \in(0,1)$ such that $F_{X_{n}, X_{n+1}}(k t) \geq F_{X_{n-1}}, x_{n}(t)$ for all $t \geq 0$ and $\mathrm{n}=1,2,3, \ldots$, then $\left\{\mathrm{x}_{\mathrm{n}}\right\}$ is a Cauchy sequence in $\mathrm{X}$.

Lemma 2.3. [11] Let $(X, F, t)$ be a Menger space. If there exists a constant $k \in(0,1)$ such that

$$
F_{x, y}(k t) \geq F_{x, y}(t) \text { for all } x, y \in X \text { and } t>0 \text {, then } x=y \text {. }
$$

A class of implicit relation. Let $\Phi$ be the set of all real continuous functions $\phi:\left(\mathrm{R}^{+}\right)^{4} \rightarrow \mathrm{R}$, non-decreasing in the first argument with the property :

a. $\quad$ For $\mathrm{u}, \mathrm{v} \geq 0, \phi(\mathrm{u}, \mathrm{v}, \mathrm{v}, \mathrm{u}) \geq 0$ or $\phi(\mathrm{u}, \mathrm{v}, \mathrm{u}, \mathrm{v}) \geq 0$ implies that $\mathrm{u} \geq \mathrm{v}$.

b. $\quad \phi(\mathrm{u}, \mathrm{u}, 1,1) \geq 0$ implies that $\mathrm{u} \geq 1$.

Example 2.3. Define $\phi\left(\mathrm{t}_{1}, \mathrm{t}_{2}, \mathrm{t}_{3}, \mathrm{t}_{4}\right)=18 \mathrm{t}_{1}-16 \mathrm{t}_{2}+8 \mathrm{t}_{3}-10 \mathrm{t}_{4}$. Then $\phi \in \Phi$.

\section{Main Result}

Theorem 3.1. Let A, B, L, M, S and T be self mappings on a complete Menger space (X, F, t) with $\mathrm{t}(\mathrm{a}, \mathrm{a}) \geq \mathrm{a}$, for some $\mathrm{a} \in[0,1]$, satisfying :

(3.1.1) $\mathrm{L}(\mathrm{X}) \subseteq \mathrm{ST}(\mathrm{X}), \mathrm{M}(\mathrm{X}) \subseteq \mathrm{AB}(\mathrm{X})$;

(3.1.2) $\mathrm{ST}(\mathrm{X})$ and $\mathrm{AB}(\mathrm{X})$ are complete subspace of $\mathrm{X}$;

(3.1.3) either $\mathrm{AB}$ or $\mathrm{L}$ is continuous;

(3.1.4) (L, AB) is compatible maps of type ( $\beta$ ) and (M, ST) is occasionally weak compatible;

(3.1.5) for some $\phi \in \Phi$, there exists $\mathrm{k} \in(0,1)$ such that for all $\mathrm{x}, \mathrm{y} \in \mathrm{X}$ and $\mathrm{t}>0$,

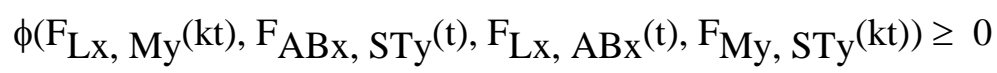

then $\mathrm{A}, \mathrm{B}, \mathrm{L}, \mathrm{M}, \mathrm{S}$ and $\mathrm{T}$ have a unique common fixed point in $\mathrm{X}$.

Proof. Let $\mathrm{x}_{0} \in X$. From condition (3.1.1) $\exists \mathrm{x}_{1}, \mathrm{x}_{2} \in X$ such that 


$$
\mathrm{Lx}_{0}=\mathrm{STx}_{1}=\mathrm{y}_{0} \text { and } \mathrm{Mx}_{1}=\mathrm{ABx}_{2}=\mathrm{y}_{1} .
$$

Inductively, we can construct sequences $\left\{x_{n}\right\}$ and $\left\{y_{n}\right\}$ in $X$ such that

$$
\begin{aligned}
\mathrm{Lx}_{2 \mathrm{n}}=\mathrm{STx}_{2 \mathrm{n}+1}=\mathrm{y}_{2 \mathrm{n}} \quad \text { and } \quad \mathrm{Mx}_{2 \mathrm{n}+1}=\mathrm{ABx}_{2 \mathrm{n}+2}=\mathrm{y}_{2 \mathrm{n}+1} & \\
& \text { for } \mathrm{n}=0,1,2, \ldots .
\end{aligned}
$$

Step 1. Putting $x=x_{2 n}$ and $y=x_{2 n+1}$ in (3.1.5), we get

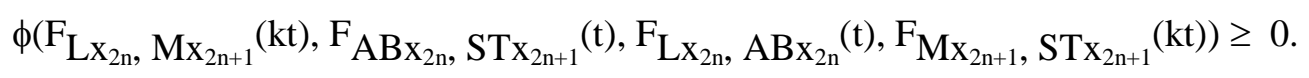

Letting $\mathrm{n} \rightarrow \infty$, we get

$$
\phi\left(F_{y_{2 n}, y_{2 n+1}}(k t), F_{y_{2 n-1}, y_{2 n}}(t), F_{y_{2 n}, y_{2 n-1}}(t), F_{y_{2 n+1}, y_{2 n}}(k t)\right) \geq 0
$$

Using (a), we get

$$
\mathrm{F}_{\mathrm{y}_{2 \mathrm{n}}, \mathrm{y}_{2 \mathrm{n}+1}}(\mathrm{kt}) \geq \mathrm{F}_{\mathrm{y}_{2 \mathrm{n}-1}, \mathrm{y}_{2 \mathrm{n}}}(\mathrm{t}) \text {. }
$$

Therefore, for all $\mathrm{n}$ even or odd, we have

$$
\mathrm{F}_{\mathrm{y}_{\mathrm{n}}, \mathrm{y}_{\mathrm{n}+1}}(\mathrm{kt}) \geq \mathrm{F}_{\mathrm{y}_{\mathrm{n}-1}, \mathrm{y}_{\mathrm{n}}}(\mathrm{t}) \text {. }
$$

Therefore, by lemma $2.1,\left\{y_{n}\right\}$ is a Cauchy sequence in $\mathrm{X}$, which is complete.

Hence $\left\{\mathrm{y}_{\mathrm{n}}\right\} \rightarrow \mathrm{z} \in \mathrm{X}$. Also its subsequences converges as follows :

$$
\left\{\mathrm{Lx}_{2 \mathrm{n}}\right\} \rightarrow \mathrm{z}, \quad\left\{\mathrm{ABx}_{2 \mathrm{n}}\right\} \rightarrow \mathrm{z}, \quad\left\{\mathrm{Mx}_{2 \mathrm{n}+1}\right\} \rightarrow \mathrm{z} \text { and }\left\{\mathrm{STx}_{2 \mathrm{n}+1}\right\} \rightarrow \mathrm{z} .
$$

\section{Case I. When $\mathrm{AB}$ is continuous.}

As $\mathrm{AB}$ is continuous, $(\mathrm{AB})^{2} \mathrm{x}_{2 \mathrm{n}} \rightarrow \mathrm{ABz}$ and $(\mathrm{AB}) \mathrm{Lx}_{2 \mathrm{n}} \rightarrow \mathrm{ABz}$.

As $(L, A B)$ is compatible pair of type $(\beta)$, so

$$
\mathrm{LLx}_{2 \mathrm{n}} \rightarrow(\mathrm{AB})(\mathrm{AB}) \mathrm{x}_{2 \mathrm{n}} \text { and so } \mathrm{LABx}_{2 \mathrm{n}} \rightarrow \mathrm{ABz}
$$

Step 2. Putting $x=A x_{2 n}$ and $y=x_{2 n+1}$ in (3.1.5), we get



Letting $\mathrm{n} \rightarrow \infty$, we get

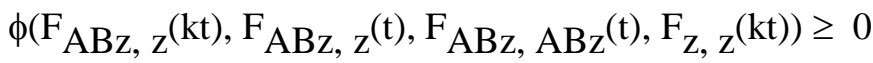

$$
\begin{aligned}
& \phi\left(\mathrm{F}_{\mathrm{ABz}, \mathrm{z}}(\mathrm{kt}), \mathrm{F}_{\mathrm{ABz}, \mathrm{z}}(\mathrm{t}), 1,1\right) \geq 0 .
\end{aligned}
$$

As $\phi$ is non-decreasing in the first argument, we have

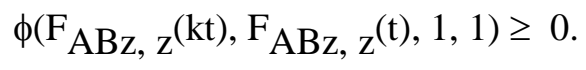

Using (b), we get

$$
\mathrm{F}_{\mathrm{ABz}, \mathrm{z}}(\mathrm{t})=1 \text {, for all } \mathrm{t}>0 \text {, }
$$

i.e. $\quad \mathrm{ABz}=\mathrm{z}$.

Step 3. Putting $x=z$ and $y=x_{2 n+1}$ in (3.1.5), we get

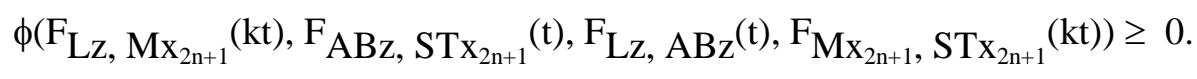


Letting $\mathrm{n} \rightarrow \infty$, we get

$$
\begin{aligned}
& \phi\left(\mathrm{F}_{\mathrm{Lz}, \mathrm{z}}(\mathrm{kt}), \mathrm{F}_{\mathrm{ABz}, \mathrm{z}}(\mathrm{t}), \mathrm{F}_{\mathrm{Lz}, A B z}(\mathrm{t}), \mathrm{F}_{\mathrm{Z}, \mathrm{z}}(\mathrm{kt})\right) \geq 0 \\
& \phi\left(\mathrm{F}_{\mathrm{Lz}, \mathrm{z}}(\mathrm{kt}), 1, \mathrm{~F}_{\mathrm{Lz}, \mathrm{z}}(\mathrm{t}), 1\right) \geq 0 .
\end{aligned}
$$

As $\phi$ is non-decreasing in the first argument, we have

$$
\phi\left(\mathrm{F}_{\mathrm{Lz}, \mathrm{z}}(\mathrm{kt}), 1, \mathrm{~F}_{\mathrm{Lz}, \mathrm{z}}(\mathrm{t}), 1\right) \geq 0 .
$$

Using (a), we get

$$
\mathrm{F}_{\mathrm{z}, \mathrm{Lz}}(\mathrm{kt})=1 \text {, for all } \mathrm{t}>0
$$

i.e. $\quad \mathrm{z}=\mathrm{Lz}$.

Thus, we have $\mathrm{z}=\mathrm{Lz}=\mathrm{ABz}$.

Step 4. Putting $x=B z$ and $y=x_{2 n+1}$ in (3.1.5), we get



Letting $\mathrm{n} \rightarrow \infty$, we get

$$
\begin{aligned}
& \phi\left(\mathrm{F}_{\mathrm{Bz}, \mathrm{z}}(\mathrm{kt}), \mathrm{F}_{\mathrm{Bz}, \mathrm{z}}(\mathrm{t}), \mathrm{F}_{\mathrm{Bz}, \mathrm{Bz}}(\mathrm{t}), \mathrm{F}_{\mathrm{z}, \mathrm{z}}(\mathrm{kt})\right) \geq 0 \\
& \phi\left(\mathrm{F}_{\mathrm{Bz}, \mathrm{z}}(\mathrm{kt}), \mathrm{F}_{\mathrm{Bz}, \mathrm{z}}(\mathrm{t}), 1,1\right) \geq 0 .
\end{aligned}
$$

As $\phi$ is non-decreasing in the first argument, we have

$$
\phi\left(\mathrm{F}_{\mathrm{Bz}, \mathrm{z}}(\mathrm{t}), \mathrm{F}_{\mathrm{Bz}, \mathrm{z}}(\mathrm{t}), 1,1\right) \geq 0 .
$$

Using (b), we have

$$
\mathrm{F}_{\mathrm{Bz}, \mathrm{z}}(\mathrm{t})=1 \text {, for all } \mathrm{t}>0 \text {, }
$$

i.e. $\quad z=B z$.

Since $\mathrm{z}=\mathrm{ABz}$, we also have

$$
\mathrm{z}=\mathrm{Az} \text {. }
$$

Therefore, $\mathrm{z}=\mathrm{Az}=\mathrm{Bz}=\mathrm{Lz}$.

Step 5. As $L(X) \subseteq S T(X)$, there exists $v \in X$ such that

$$
\mathrm{z}=\mathrm{Lz}=\mathrm{STv} .
$$

Putting $x=x_{2 n}$ and $y=v$ in (3.1.5), we get



Letting $\mathrm{n} \rightarrow \infty$, we get

$$
\begin{aligned}
& \phi\left(\mathrm{F}_{\mathrm{z}, \mathrm{Mv}}(\mathrm{kt}), \mathrm{F}_{\mathrm{z}, \mathrm{STv}}(\mathrm{t}), \mathrm{F}_{\mathrm{z}, \mathrm{z}}(\mathrm{t}), \mathrm{F}_{\mathrm{Mv}, \mathrm{z}}(\mathrm{kt})\right) \geq 0 \\
& \phi\left(\mathrm{F}_{\mathrm{Z}, \mathrm{Mv}}(\mathrm{kt}), 1,1, \mathrm{~F}_{\mathrm{Z}, \mathrm{Mv}}(\mathrm{kt})\right) \geq 0
\end{aligned}
$$

Using (a), we have

$$
\mathrm{F}_{\mathrm{Z}, \mathrm{Mv}}(\mathrm{kt}) \geq 1, \text { for all } \mathrm{t}>0 \text {. }
$$

Hence, $\mathrm{F}_{\mathrm{Z}, \mathrm{Mv}}(\mathrm{t})=1$. 
Thus, $\mathrm{z}=\mathrm{Mv}$.

Therefore, $\mathrm{z}=\mathrm{Mv}=\mathrm{ST}$.

As (M, ST) is occasionally weakly compatible, we have

$$
\text { STMv }=\text { MSTv. Thus, STz }=\text { Mz. }
$$

Step 6. Putting $x=x_{2 n}$ and $y=z$ in (3.1.5), we get

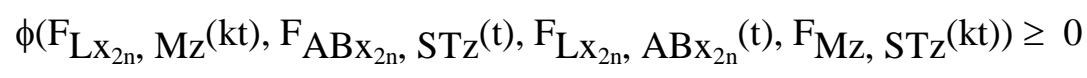

Letting $\mathrm{n} \rightarrow \infty$, we get

$$
\phi\left(\mathrm{F}_{\mathrm{z}, \mathrm{Mz}}(\mathrm{kt}), \mathrm{F}_{\mathrm{Z}, \mathrm{Mz}}(\mathrm{t}), 1,1\right) \geq 0 .
$$

As $\phi$ is non-decreasing in the first argument, we have

$$
\phi\left(\mathrm{F}_{\mathrm{Z}, \mathrm{Mz}}(\mathrm{t}), \mathrm{F}_{\mathrm{Z}, \mathrm{Mz}}(\mathrm{t}), 1,1\right) \geq 0 .
$$

Using (b), we have

$$
\mathrm{F}_{\mathrm{z}, \mathrm{Mz}}(\mathrm{t}) \geq 1, \text { for all } \mathrm{t}>0 \text {. }
$$

Thus, $\mathrm{F}_{\mathrm{Z}, \mathrm{Mz}}(\mathrm{t})=1$, we have

$$
\mathrm{z}=\mathrm{Mz}=\mathrm{STz} \text {. }
$$

Step 7. Putting $\mathrm{x}=\mathrm{x}_{2 \mathrm{n}}$ and $\mathrm{y}=\mathrm{Tz}$ in (3.1.5) and using Step 5, we get

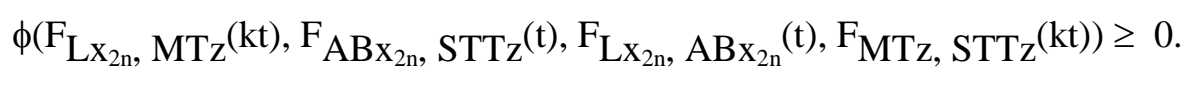

Letting $\mathrm{n} \rightarrow \infty$, we get

$$
\begin{aligned}
& \phi\left(\mathrm{F}_{\mathrm{Lz}, T z}(\mathrm{kt}), \mathrm{F}_{\mathrm{z}, \mathrm{Tz}}(\mathrm{t}), \mathrm{F}_{\mathrm{z}, \mathrm{z}}(\mathrm{t}), \mathrm{F}_{\mathrm{Tz}, T z}(\mathrm{kt})\right) \geq 0 \\
& \phi\left(\mathrm{F}_{\mathrm{Z}, \mathrm{Tz}}(\mathrm{kt}), \mathrm{F}_{\mathrm{Z}, \mathrm{Tz}}(\mathrm{t}), 1,1\right) \geq 0 .
\end{aligned}
$$

As $\phi$ is non-decreasing in the first argument, we have

$$
\phi\left(\mathrm{F}_{\mathrm{z}, \mathrm{Tz}}(\mathrm{t}), \mathrm{F}_{\mathrm{z}, \mathrm{Tz}}(\mathrm{t}), 1,1\right) \geq 0 .
$$

Using (b), we have

$$
\mathrm{F}_{\mathrm{Z}, \mathrm{Tz}}(\mathrm{t}) \geq 1 \text {, for all } \mathrm{t}>0 \text {. }
$$

Thus, $\mathrm{F}_{\mathrm{z}, \mathrm{Tz}}(\mathrm{t})=1$, we have

$$
\mathrm{z}=\mathrm{Tz} \text {. }
$$

Since $\mathrm{Tz}=\mathrm{STz}$, we also have $\mathrm{z}=\mathrm{Sz}$.

Hence

$$
\mathrm{Az}=\mathrm{Bz}=\mathrm{Lz}=\mathrm{Mz}=\mathrm{Tz}=\mathrm{Sz}=\mathrm{z} .
$$

Hence, the six self maps have a common fixed point in this case.

\section{Case II. When $L$ is continuous}

As $\mathrm{L}$ is continuous, $\mathrm{L}^{2} \mathrm{x}_{2 \mathrm{n}} \rightarrow \mathrm{Lz}$ and $\mathrm{L}(\mathrm{AB}) \mathrm{x}_{2 \mathrm{n}} \rightarrow \mathrm{Lz}$.

As $(L, A B)$ is compatible map of type $(\beta)$, so 


$$
\mathrm{LLx}_{2 \mathrm{n}} \rightarrow(\mathrm{AB})(\mathrm{AB}) \mathrm{x}_{2 \mathrm{n}} \text { and } \mathrm{LABx}_{2 \mathrm{n}} \rightarrow \mathrm{ABz}
$$

By uniqueness of limit in Menger space, we have

$$
\mathrm{Lz}=\mathrm{ABz} .
$$

Step 8. Putting $x=z$ and $y=x_{2 n+1}$ in (3.1.5), we get

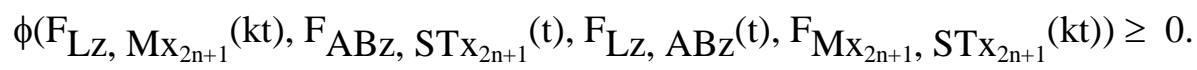

Letting $\mathrm{n} \rightarrow \infty$, we get

$$
\begin{aligned}
& \phi\left(\mathrm{F}_{\mathrm{Lz}, \mathrm{z}}(\mathrm{kt}), \mathrm{F}_{\mathrm{Lz}, \mathrm{z}}(\mathrm{t}), \mathrm{F}_{\mathrm{Lz}, \mathrm{Lz}}(\mathrm{t}), \mathrm{F}_{\mathrm{z}, \mathrm{z}}(\mathrm{kt})\right) \geq 0 \\
& \phi\left(\mathrm{F}_{\mathrm{Lz}, \mathrm{z}}(\mathrm{kt}), \mathrm{F}_{\mathrm{Lz}, \mathrm{z}}(\mathrm{t}), 1,1\right) \geq 0 .
\end{aligned}
$$

As $\phi$ is non-decreasing in the first argument, we have

$$
\phi\left(\mathrm{F}_{\mathrm{Lz}, \mathrm{z}}(\mathrm{t}), \mathrm{F}_{\mathrm{Lz}, \mathrm{z}}(\mathrm{t}), 1,1\right) \geq 0 .
$$

Using (b), we have

$$
\mathrm{F}_{\mathrm{Z}, \mathrm{Lz}}(\mathrm{t}) \geq 1 \text {, for all } \mathrm{t}>0 \text {. }
$$

Thus, $\mathrm{F}_{\mathrm{z}, \mathrm{Lz}}(\mathrm{t})=1$

$\Rightarrow \quad \mathrm{z}=\mathrm{Lz}$.

Therefore,

$$
\mathrm{z}=\mathrm{Lz}=\mathrm{ABz}
$$

Step 9. Putting $x=B z$ and $y=x_{2 n+1}$ in (3.1.5), we get

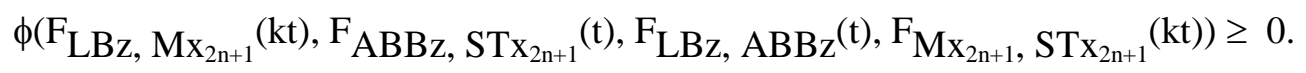

Letting $\mathrm{n} \rightarrow \infty$, we get

$$
\begin{aligned}
& \phi\left(\mathrm{F}_{\mathrm{Bz}, \mathrm{z}}(\mathrm{kt}), \mathrm{F}_{\mathrm{Bz}, \mathrm{z}}(\mathrm{t}), \mathrm{F}_{\mathrm{Bz}, \mathrm{Bz}}(\mathrm{t}), \mathrm{F}_{\mathrm{z}, \mathrm{z}}(\mathrm{kt})\right) \geq 0 \\
& \phi\left(\mathrm{F}_{\mathrm{Bz}, \mathrm{z}}(\mathrm{kt}), \mathrm{F}_{\mathrm{Bz}, \mathrm{z}}(\mathrm{t}), 1,1\right) \geq 0 .
\end{aligned}
$$

As $\phi$ is non-decreasing in the first argument, we have

$$
\phi\left(\mathrm{F}_{\mathrm{Bz}, \mathrm{z}}(\mathrm{t}), \mathrm{F}_{\mathrm{Bz}, \mathrm{z}}(\mathrm{t}), 1,1\right) \geq 0 .
$$

Using (b), we have

$$
\mathrm{F}_{\mathrm{Bz}, \mathrm{z}}(\mathrm{t}) \geq 1 \text {, for all } \mathrm{t}>0 \text {. }
$$

Thus, $\mathrm{F}_{\mathrm{Bz}, \mathrm{z}}(\mathrm{t})=1$

$\Rightarrow \quad \mathrm{z}=\mathrm{Bz}$.

Since $\mathrm{z}=\mathrm{ABz}$, we also have $\mathrm{z}=\mathrm{Az}$.

Therefore, $\quad \mathrm{z}=\mathrm{Az}=\mathrm{Bz}=\mathrm{Lz}$.

Step 10. As $L(X) \subseteq S T(X)$, there exists $v \in X$ such that

$$
\mathrm{z}=\mathrm{Lz}=\mathrm{STv} .
$$

Putting $x=x_{2 n}$ and $y=v$ in (3.1.5), we get 


$$
\phi\left(\mathrm{F}_{\mathrm{Lx}_{2 \mathrm{n}}, \mathrm{Mv}}(\mathrm{kt}), \mathrm{F}_{\mathrm{ABx}_{2 \mathrm{n}}, \mathrm{STv}_{\mathrm{v}}(\mathrm{t}), \mathrm{F}_{\mathrm{Lx}_{2 \mathrm{n}}, \mathrm{ABx}_{2 \mathrm{n}}}(\mathrm{t}), \mathrm{F}_{\mathrm{Mv}}, \mathrm{STv}}(\mathrm{kt})\right) \geq 0 .
$$

Letting $\mathrm{n} \rightarrow \infty$, we get

$$
\begin{aligned}
& \phi\left(\mathrm{F}_{\mathrm{z}, \mathrm{Mv}}(\mathrm{kt}), \mathrm{F}_{\mathrm{z}, \operatorname{STv}}(\mathrm{t}), \mathrm{F}_{\mathrm{z}, \mathrm{z}}(\mathrm{t}), \mathrm{F}_{\mathrm{Mv}, \mathrm{z}}(\mathrm{kt})\right) \geq 0 \\
& \phi\left(\mathrm{F}_{\mathrm{z}, \mathrm{Mv}}(\mathrm{kt}), 1,1, \mathrm{~F}_{\mathrm{z}, \mathrm{Mv}}(\mathrm{kt})\right) \geq 0
\end{aligned}
$$

Using (a), we have

$$
\mathrm{F}_{\mathrm{z}, \mathrm{Mv}}(\mathrm{kt}) \geq 1, \text { for all } \mathrm{t}>0 \text {. }
$$

Hence, $\mathrm{F}_{\mathrm{Z}, \mathrm{Mv}}(\mathrm{t})=1$.

Thus, $\mathrm{z}=\mathrm{Mv}$.

Therefore, $\mathrm{z}=\mathrm{Mv}=\mathrm{ST}$.

As (M, ST) is occasionally weakly compatible, we have

$$
\mathrm{STMv}=\text { MSTv. }
$$

Thus, $\mathrm{STz}=\mathrm{Mz}$.

Step 11. Putting $x=x_{2 n}$ and $y=z$ in (3.1.5), we get

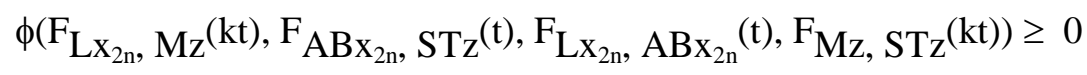

Letting $\mathrm{n} \rightarrow \infty$, we get

$$
\phi\left(\mathrm{F}_{\mathrm{z}, \mathrm{Mz}}(\mathrm{kt}), \mathrm{F}_{\mathrm{z}, \mathrm{Mz}}(\mathrm{t}), 1,1\right) \geq 0 .
$$

As $\phi$ is non-decreasing in the first argument, we have

$$
\phi\left(\mathrm{F}_{\mathrm{Z}, \mathrm{Mz}}(\mathrm{t}), \mathrm{F}_{\mathrm{Z}, \mathrm{Mz}}(\mathrm{t}), 1,1\right) \geq 0 .
$$

Using (b), we have

$$
\mathrm{F}_{\mathrm{z}, \mathrm{Mz}}(\mathrm{t}) \geq 1, \text { for all } \mathrm{t}>0 \text {. }
$$

Thus, $\mathrm{F}_{\mathrm{z}, \mathrm{Mz}}(\mathrm{t})=1$, we have

$$
\mathrm{z}=\mathrm{Mz}=\mathrm{STz} \text {. }
$$

Step 12. Putting $x=x_{2 n}$ and $y=T z$ in (3.1.5) and using Step 5, we get

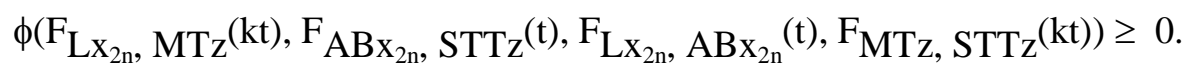

Letting $\mathrm{n} \rightarrow \infty$, we get

$$
\begin{aligned}
& \phi\left(\mathrm{F}_{\mathrm{Lz}, T z}(\mathrm{kt}), \mathrm{F}_{\mathrm{z}, \mathrm{Tz}}(\mathrm{t}), \mathrm{F}_{\mathrm{Z}, \mathrm{z}}(\mathrm{t}), \mathrm{F}_{\mathrm{Tz}, \mathrm{Tz}}(\mathrm{kt})\right) \geq 0 \\
& \phi\left(\mathrm{F}_{\mathrm{z}, \mathrm{Tz}}(\mathrm{kt}), \mathrm{F}_{\mathrm{Z}, \mathrm{Tz}}(\mathrm{t}), 1,1\right) \geq 0 .
\end{aligned}
$$

As $\phi$ is non-decreasing in the first argument, we have

$$
\phi\left(\mathrm{F}_{\mathrm{Z}, \mathrm{Tz}}(\mathrm{kt}), \mathrm{F}_{\mathrm{z}, \mathrm{Tz}}(\mathrm{t}), 1,1\right) \geq 0 .
$$

Using (b), we have

$$
\mathrm{F}_{\mathrm{Z}, \mathrm{Tz}}(\mathrm{t}) \geq 1 \text {, for all } \mathrm{t}>0 \text {. }
$$


Thus, $\mathrm{F}_{\mathrm{z}, \mathrm{Tz}}(\mathrm{t})=1$, we have

$$
\mathrm{z}=\mathrm{Tz} \text {. }
$$

Since $\mathrm{Tz}=\mathrm{STz}$, we also have $\mathrm{z}=\mathrm{Sz}$.

Hence $\mathrm{Az}=\mathrm{Bz}=\mathrm{Lz}=\mathrm{Mz}=\mathrm{Tz}=\mathrm{Sz}=\mathrm{z}$.

Hence, the six self maps have a common fixed point in this case also.

Uniqueness. Let $\mathrm{w}$ be another common fixed point of $\mathrm{A}, \mathrm{B}, \mathrm{L}, \mathrm{M}, \mathrm{S}$ and $\mathrm{T}$; then $\mathrm{w}=\mathrm{Aw}=\mathrm{Bw}=\mathrm{Lw}=\mathrm{Mw}=\mathrm{Sw}=\mathrm{Tw}$.

$$
\begin{aligned}
& \text { Putting } \mathrm{x}=\mathrm{z} \text { and } \mathrm{y}=\mathrm{w} \text { in (3.1.5), we get }
\end{aligned}
$$

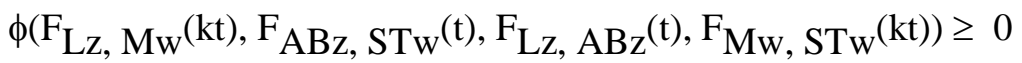

$$
\begin{aligned}
& \phi\left(\mathrm{F}_{\mathrm{z}, \mathrm{w}}(\mathrm{kt}), \mathrm{F}_{\mathrm{z}, \mathrm{w}}(\mathrm{t}), \mathrm{F}_{\mathrm{z}, \mathrm{z}}(\mathrm{t}), \mathrm{F}_{\mathrm{w}, \mathrm{w}}(\mathrm{kt})\right) \geq 0 \\
& \phi\left(\mathrm{F}_{\mathrm{z}, \mathrm{w}}(\mathrm{kt}), \mathrm{F}_{\mathrm{z}, \mathrm{w}}(\mathrm{t}), 1,1\right) \geq 0 \text {. }
\end{aligned}
$$

As $\phi$ is non-decreasing in the first argument, we have

$$
\phi\left(\mathrm{F}_{\mathrm{z}, \mathrm{w}}(\mathrm{t}), \mathrm{F}_{\mathrm{z}, \mathrm{w}}(\mathrm{t}), 1,1\right) \geq 0 .
$$

Using (b), we have

$$
\mathrm{F}_{\mathrm{z}, \mathrm{w}}(\mathrm{t}) \geq 1, \text { for all } \mathrm{t}>0 \text {. }
$$

Thus, $\mathrm{F}_{\mathrm{Z}, \mathrm{w}}(\mathrm{t})=1$,

i.e., $\quad z=w$.

Therefore, $\mathrm{z}$ is a unique common fixed point of $\mathrm{A}, \mathrm{B}, \mathrm{L}, \mathrm{M}, \mathrm{S} \& \mathrm{~T}$.

This completes the proof.

Remark 3.1. The above theorem is a generalization of the result of Pant et. al. [7] in the sense that the conditions of semi-compatibility and weak compatibility have been replaced by compatibility of type $(\beta)$ and occasionally weakly compatible.

On taking $\mathrm{B}=\mathrm{T}=\mathrm{I}$ (the identity map) on $\mathrm{X}$ in theorem 3.1, we get the following corollary.

Corollary 3.1. Let A, L, M, and $\mathrm{S}$ be self mappings on a complete Menger space $(X, F, t)$ with $t(a, a) \geq a$, for some a $\in[0,1]$, satisfying :

(3.1.1) $\mathrm{L}(\mathrm{X}) \subseteq \mathrm{S}(\mathrm{X}), \mathrm{M}(\mathrm{X}) \subseteq \mathrm{A}(\mathrm{X})$

(3.1.2) $\mathrm{S}(\mathrm{X})$ and $\mathrm{A}(\mathrm{X})$ are complete subspace of $\mathrm{X}$;

(3.1.3) either A or L is continuous;

(3.1.4) (L, A) is compatible maps of type $(\beta)$ and $(\mathrm{M}, \mathrm{S})$ is occasionally weak compatible;

(3.1.5) for some $\phi \in \Phi$, there exists $\mathrm{k} \in(0,1)$ such that for all $\mathrm{x}, \mathrm{y} \in \mathrm{X}$ and $\mathrm{t}>0$,



then $\mathrm{A}, \mathrm{B}, \mathrm{L}, \mathrm{M}, \mathrm{S}$ and $\mathrm{T}$ have a unique common fixed point in $\mathrm{X}$.

\section{REFERENCES}

[1] Cho, Y.J., Murthy, P.P. and Stojakovik, M., Compatible mappings of type (A) and common fixed point in Menger space, Comm. Korean Math. Soc. 7 (2), (1992), 325-339. 
[2] Jain, A. and Chaudhary, B., On common fixed point theorems for semi-compatible and occasionally weakly compatible mappings in Menger space, International Journal of Research and Reviews in Applied Sciences, Vol. 14 (3), (2013), 662-670.

[3] Jungck, G., Compatible mappings and common fixed points, Internat. J. Math. and Math. Sci. 9(4), (1986), 771-779.

[4] Jungck, G. and Rhoades, B.E., Fixed points for set valued functions without continuity, Indian J. Pure Appl. Math. 29(1998), 227-238.

[5] Menger, K., Statistical metrics, Proc. Nat. Acad. Sci. USA. 28(1942), 535 -537.

[6] Mishra, S.N., Common fixed points of compatible mappings in PM-spaces, Math. Japon. 36(2), (1991), 283-289.

[7] Pant, B.D. and Chauhan, S., Common fixed point theorems for semi-compatible mappings using implicit relation, Int. Journal of Math. Analysis, 3 (28), (2009), 1389-1398.

[8] Schweizer, B. and Sklar, A., Statistical metric spaces, Pacific J. Math. 10 (1960), 313-334.

[9] Sehgal, V.M. and Bharucha-Reid, A.T., Fixed points of contraction maps on probabilistic metric spaces, Math. System Theory 6(1972), 97- 102.

[10] Sessa, S., On a weak commutativity condition of mappings in fixed point consideration, Publ. Inst. Math. Beograd 32(46), (1982), 146-153.

[11] Singh, S.L. and Pant, B.D., Common fixed point theorems in probabilistic metric spaces and extension to uniform spaces, Honam. Math. J. 6 (1984), 1-12.

\section{AUTHORS' BIOGRAPHY}
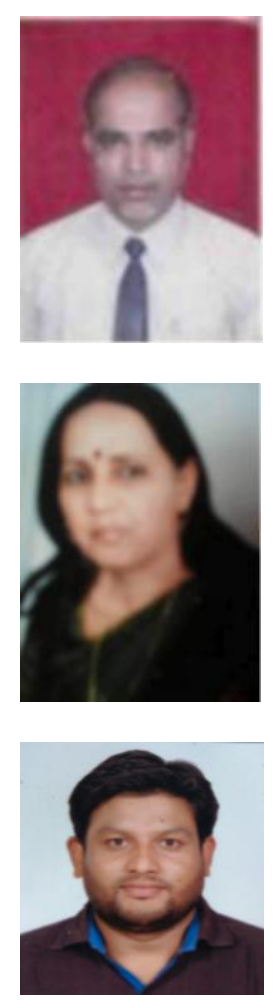

Dr. V. H. Badshah, presently working on the post of Professor and Head, School of Studies in Mathematics, Vikram University, Ujjain. He has 27 years of teaching experience. He has selected on the post of Scientific Assistance in the Indian Institute of Geo-Magnetism Colaba, Mumbai. His area of interest includes Functional Analysis, Fixed Point Theory, Mathematical Modeling and Operations Research. He has authored many books. Many students have got Ph.D. under his supervision.

Dr. Suman Jain, presently working of the post of Professor and Head, Department of Mathematics, Govt. College Kalapipal (M.P.). She has 27 years of teaching experience. Her areas of interest includes fixed point theory and Mathematical Modeling. Many students have got Ph.D. under her supervision.

Mr. Subhash Mandloi, did his Post Graduation in Mathematics in the year 2008 and M.Phil. (Mathmatics) in 2009 from School of Studies in Mathematics, Vikram University, Ujjain. He has a teaching experience of 8 years. 